\title{
Optimal solution error covariance in highly nonlinear problems of variational data assimilation
}

\author{
V. Shutyaev ${ }^{1}$, I. Gejadze ${ }^{2}$, G. J. M. Copeland ${ }^{2}$, and F.-X. Le Dimet ${ }^{3}$ \\ ${ }^{1}$ Institute of Numerical Mathematics, Russian Academy of Sciences, 119333 Gubkina 8, Moscow, Russia \\ ${ }^{2}$ Department of Civil Engineering, University of Strathclyde, 107 Rottenrow, Glasgow, G4 ONG, UK \\ ${ }^{3}$ MOISE project (CNRS, INRIA, UJF, INPG), LJK, Université de Grenoble, BP 53, 38041 Grenoble, France
}

Correspondence to: V. Shutyaev (shutyaev@inm.ras.ru)

Received: 6 July 2011 - Revised: 16 February 2012 - Accepted: 20 February 2012 - Published: 16 March 2012

\begin{abstract}
The problem of variational data assimilation (DA) for a nonlinear evolution model is formulated as an optimal control problem to find the initial condition, boundary conditions and/or model parameters. The input data contain observation and background errors, hence there is an error in the optimal solution. For mildly nonlinear dynamics, the covariance matrix of the optimal solution error can be approximated by the inverse Hessian of the cost function. For problems with strongly nonlinear dynamics, a new statistical method based on the computation of a sample of inverse Hessians is suggested. This method relies on the efficient computation of the inverse Hessian by means of iterative methods (Lanczos and quasi-Newton BFGS) with preconditioning. Numerical examples are presented for the model governed by the Burgers equation with a nonlinear viscous term.
\end{abstract}

\section{Introduction}

State and/or parameter estimation for dynamical geophysical flow models is an important problem in meteorology and oceanography. Among the few methods feasible for solving these non-stationary large-scale problems, the variational data assimilation (DA) method, called "4D-Var", is the preferred method implemented at some major operational centers (e.g. Courtier et al., 1994; Fisher et al., 2009). From the mathematical point of view, these problems can be formulated as optimal control problems (e.g. Lions, 1986; Le Dimet and Talagrand, 1986) to find unknown control variables in such a way that a cost function related to the observation and a priori data takes its minimum value. A necessary optimality condition leads to the so-called optimality system, which contains all the available information and involves the original and adjoint models. Due to the input errors (background and observation errors), there is an error in the op- timal solution. Its statistical properties are very important for quantifying the accuracy of the optimal solution (which is necessary to evaluate the quality of the forecast), for sequential variational state estimation and optimal design of observation schemes. Assuming that the probability density function (p.d.f.) of the optimal solution error can be reasonably approximated by the normal (Gaussian) distribution, the optimal solution error covariance matrix (referred to below simply as "covariance") is its most important statistic to be estimated. If the errors of the input data are random and normally distributed, then for a linearized finite-dimensional error evolution model, the covariance is given by the inverse Hessian of the cost function (e.g. Thacker, 1989; Rabier and Courtier, 1992). This is an extension of a well-known result from nonlinear regression (Draper and Smith, 1981) to the case of nonlinear dynamical systems. A similar result in the continuous case was presented by Gejadze et al. (2008). In terms of continuous representation, it is said that the covariance operator can be approximated by the inverse Hessian of the auxiliary control problem based on the tangent linear model (TLM) constraints, if the so-called tangent linear hypothesis (TLH) is valid. The TLH implies that the error dynamics can be satisfactorily described by the TLM. It was demonstrated by Gejadze et al. $(2010,2011)$ that approximation of the covariance by the inverse Hessian could be sometimes sufficiently accurate even though the TLH is not valid. However, in the case of highly nonlinear dynamics such an approximation may not be valid at all (see, for example, Pires et al., 1996). In the present paper, for the case under consideration, we do the following: (a) present an argument that even in this case the p.d.f. of the optimal solution error may still be represented by a normal distribution defined by the covariance matrix; (b) outline a new method for estimation of the covariance; (c) discuss implementation potentially feasible for large-scale dynamical models. One of the objectives of this paper is to highlight the concept of 
the Effective Inverse Hessian (EIH), first introduced by Gejadze et al. (2011), to the geophysical research community. The closest concept to this is probably the Expected Fisher Information Matrix used in Bayesian estimation theory.

\section{Statement of the problem}

Consider the mathematical model of a physical process that is described by the evolution problem

$$
\left\{\begin{aligned}
\frac{\partial \varphi}{\partial t} & =F(\varphi), \quad t \in(0, T) \\
\left.\varphi\right|_{t=0} & =u,
\end{aligned}\right.
$$

where $\varphi=\varphi(t)$ is the unknown function belonging for any $t$ to a Hilbert space $X, u \in X, F$ is a nonlinear operator mapping $X$ into $X$. Let $Y=L_{2}(0, T ; X)$ be a space of abstract functions $\varphi(t)$ with values in $X$, with the norm $\|\varphi\|=\left(\int_{0}^{T}\|\varphi\|_{X}^{2} d t\right)^{1 / 2}$. Suppose that for a given $u \in X$ there exists a unique solution $\varphi \in Y$ to Eq. (1).

Let $\bar{u}$ be the "exact" initial state and $\bar{\varphi}-$ the solution to the problem Eq. (1) with $u=\bar{u}$, i.e. the "exact" state evolution. We define the input data as follows: the background function $u_{\mathrm{b}} \in X, u_{\mathrm{b}}=\bar{u}+\xi_{\mathrm{b}}$ and the observations $y \in Y_{\mathrm{o}}$, $y=C \bar{\varphi}+\xi_{\mathrm{o}}$, where $C: Y \rightarrow Y_{o}$ is a linear bounded operator (observation operator) and $Y_{O}$ is a Hilbert space (observation space), $\xi_{\mathrm{b}} \in X, \xi_{\mathrm{o}} \in Y_{o}$. In particular, $Y_{o}$ may be finite-dimensional (both in space and in time). The random variables $\xi_{\mathrm{b}}$ and $\xi_{\mathrm{o}}$ may be regarded as the background and the observation error, respectively. Assuming that these errors are normally distributed, unbiased and mutually uncorrelated, we define the covariance operators $V_{\mathrm{b}} \cdot=E\left[\left(\cdot, \xi_{\mathrm{b}}\right)_{X} \xi_{\mathrm{b}}\right]$ and $V_{\mathrm{o}} \cdot=E\left[\left(\cdot, \xi_{0}\right)_{Y_{o}} \xi_{0}\right]$, where "." denotes an argument of the respective operator, and $E$ is the expectation. We suppose that $V_{\mathrm{b}}$ and $V_{\mathrm{o}}$ are positive definite, hence invertible.

Let us introduce a cost function $J(u)$

$$
\begin{aligned}
& J(u)=\frac{1}{2}\left(V_{\mathrm{b}}^{-1}\left(u-u_{b}\right), u-u_{b}\right)_{X}+ \\
& +\frac{1}{2}\left(V_{\mathrm{o}}^{-1}(C \varphi-y), C \varphi-y\right)_{Y_{o}},
\end{aligned}
$$

and formulate the following DA problem (optimal control problem) with the aim to identify the initial condition: find $u \in X$ and $\varphi \in Y$ such that they satisfy Eq. (1) and the cost function $J(u)$ takes its minimum value. Further we assume that the optimal solution error $\delta u=u-\bar{u}$ is unbiased, i.e. $E[\delta u]=0$, with the covariance operator $V_{\delta u} \cdot=$ $E\left[(\cdot, \delta u)_{X} \delta u\right]$.

Let us introduce the operator $R: X \rightarrow Y_{\mathrm{o}}$ as follows

$R v=C \psi, v \in X$,

where $\psi \in Y$ is the solution of the tangent linear problem

$\left\{\begin{aligned} \frac{\partial \psi}{\partial t}-F^{\prime}(\varphi) \psi & =0, t \in(0, T), \\ \left.\psi\right|_{t=0} & =v .\end{aligned}\right.$
For a given $v$ we solve the problem Eq. (4), and then find $R v$ by Eq. (3). The definition of $R$ involves $\varphi=\bar{\varphi}+\delta \varphi$ dependent on $u=\bar{u}+\delta u$ via Eq. (1), thus we can write as follows: $R=R(\bar{u}, \delta u)$. It has been shown in (Gejadze et al., 2008) that the optimal solution error $\delta u=u-\bar{u}$ and data errors $\xi_{\mathrm{b}}$ and $\xi_{0}$ are related via the following exact operator equation

$$
\begin{aligned}
& \left(V_{\mathrm{b}}^{-1}+R^{*}(\bar{u}, \delta u) V_{\mathrm{o}}^{-1} R(\bar{u}, \tau \delta u)\right) \delta u= \\
& =V_{\mathrm{b}}^{-1} \xi_{\mathrm{b}}+R^{*}(\bar{u}, \delta u) V_{\mathrm{o}}^{-1} \xi_{\mathrm{o}},
\end{aligned}
$$

where $R^{*}$ is the adjoint to $R$ and $\tau \in[0,1]$ is a parameter chosen to make the truncated Taylor series exact.

Let $H(\bar{u})=V_{\mathrm{b}}^{-1}+R^{*}(\bar{u}, 0) V_{\mathrm{o}}^{-1} R(\bar{u}, 0)$ be the Hessian of the linearized (auxiliary) control problem (Gejadze et al., 2008). Under the hypothesis that $F$ is twice continuously Fréchet differentiable, the error Eq. (5) is approximated by:

$H(\bar{u}) \delta u=V_{\mathrm{b}}^{-1} \xi_{\mathrm{b}}+R^{*}(\bar{u}, 0) V_{\mathrm{o}}^{-1} \xi_{\mathrm{o}}$.

From Eq. (6) it is easy to see that

$V_{\delta u}=[H(\bar{u})]^{-1}$.

This is a well-established result (Courtier et al., 1994; Rabier and Courtier, 1992; Thacker, 1989), which is usually deduced (without considering Eq. 5) by straightforwardly linearizing the original nonlinear DA problem Eqs. (1)-(2) under the assumption that

$F(\varphi)-F(\bar{\varphi}) \approx F^{\prime}(\bar{\varphi}) \delta \varphi$,

which is called the "tangent linear hypothesis". It is said that $V_{\delta u}$ can be approximated by $[H(\bar{u})]^{-1}$ if the TLH Eq. (8) is valid. That usually happens if the nonlinearity is mild and/or the error $\delta u$ and, subsequently, $\delta \varphi$ are small. We derive Eq. (7) via Eq. (5). From this derivation one can see that the accuracy of Eq. (7) depends on the accuracy of the approximations $R(\bar{u}, \tau \delta u) \approx R(\bar{u}, 0)$ and $R^{*}(\bar{u}, \delta u) \approx R^{*}(\bar{u}, 0)$ in Eq. (5). Clearly, the transition from Eq. (5) to Eq. (6) could still be valid even though Eq. (8) is not satisfied.

As already mentioned, we can use formula Eq. (7) if the TLH is valid and, in some cases beyond the range of its validity. In the general case, however, one may not expect $H^{-1}(\bar{u})$ always to be a satisfactory approximation to $V_{\delta u}$. In Fig. 1 we present a specially designed example for the evolution model governed by the 1-D Burgers equation (for details see Sect. 4). The difference between the reference value of the variance (circles) and the inverse Hessian based value (bold solid line) can be clearly seen within the ellipse. The reference variance is obtained by a direct Monte Carlo simulation.

Since $R^{*}(\bar{u}, 0)$ and $H(\bar{u})$ in Eq. (6) are linear operators and we assume that errors $\xi_{\mathrm{b}}$ and $\xi_{\mathrm{o}}$ are unbiased and normally distributed, then $\delta u \sim \mathcal{N}\left(0, V_{\delta u}\right)$. Clearly, this result is valid as far as the TLH and consequently Eq. (6) itself are satisfied. However, for highly nonlinear dynamical models the TLH often breaks down (e.g. Pires et al., 1996); thus, we have to 


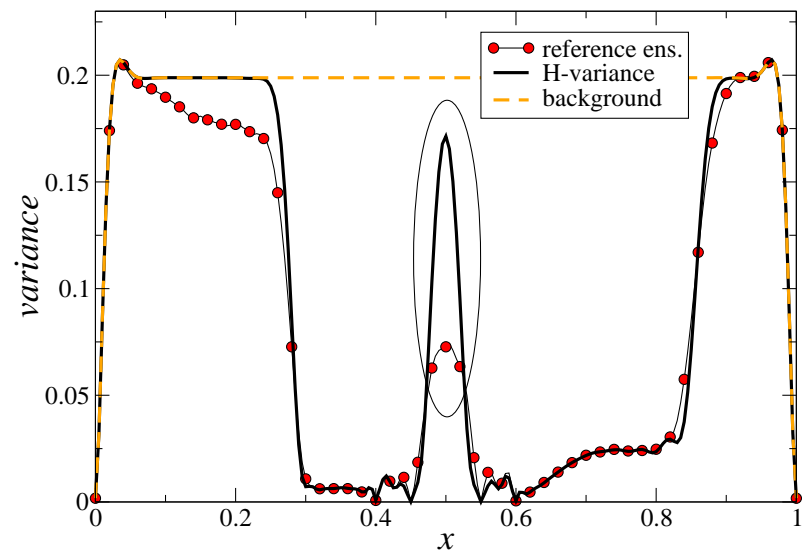

Fig. 1. Reference variance, variance by the inverse Hessian and background variance.

answer the following question: can the p.d.f. of $\delta u$ still be approximated by the normal distribution? If the answer is positive, one should look for a better approximation of the covariance than that given by Eq. (7).

Let us consider the cost function Eq. (2), but without the background term. The corresponding error equation Eq. (5) is then as follows:

$R^{*}(\bar{u}, \delta u) V_{\mathrm{o}}^{-1} R(\bar{u}, \tau \delta u) \delta u=R^{*}(\bar{u}, \delta u) V_{\mathrm{o}}^{-1} \xi_{\mathrm{o}}$.

For a univariate case, the classical result (see Jennrich, 1969) is that $\delta u$ is asymptotically normal if $\xi_{\mathrm{o}}$ is an independent identically distributed (i.i.d.) random variable with $E\left[\xi_{0}\right]=0$ and $E\left[\xi_{0}^{2}\right]=\sigma^{2}<\infty$ ("asymptotically" means that $T \rightarrow \infty$ given the finite observation time step $d t$, or $d t \rightarrow 0$ given the finite observation window $[0, T]$ ). Let us stress that for the asymptotic normality of $\delta u$, the error $\xi_{\mathrm{o}}$ is not required to be normal. This original result has been generalized to the multivariate case and to the case of dependent, yet identically distributed observations (White and Domowitz, 1984), whereas an even more general case is considered in (Yuan and Jennrich, 1998). Here we consider the complete cost function Eq. (2) and, correspondingly, the error Eq. (5), which contains terms related to the background term. To analyze a possible impact of these terms let us follow the reasoning in (Amemiya, 1983), pp. 337-345, where the error equation equivalent to Eq. (9) is derived in a slightly different form. It is concluded that the error $\delta u$ is asymptotically normal when: (a) the right-hand side of the error equation is normal; (b) the left-hand side matrix converges in probability to a non-random value. These conditions are met under certain general regularity requirements to the operator $R$, which are incomparably weaker than the TLH and do not depend on the magnitude of the input errors. Clearly, as applied to Eq. (5), the first condition holds if $\xi_{\mathrm{b}}$ is normally distributed. Since $V_{\mathrm{b}}^{-1}$ is a constant matrix, the second condition always holds as long as it holds for $R^{*}(\bar{u}, \delta u) V_{\mathrm{o}}^{-1} R(\bar{u}, \tau \delta u)$. Therefore, one may conclude that $\delta u$ from Eq. (5) is bound to remain asymptotically normal. In practice the observation window $[0, T]$ and time step $d t$ are always finite implying the finite number of i.i.d. observations. Moreover, it is not easy to assess how large the number of observations must be for the desired asymptotic properties to be reasonably approximated. Some nonlinear least-square problems, in which the normality of the estimation error holds for "practically relevant" sample sizes, are said to exhibit a "close-to-linear" statistical behavior (Ratkowsky, 1983). The method suggested in (Ratkowsky, 1983) to verify this behavior is, essentially, a normality test applied to a generated sample of optimal solutions, which is hardly feasible for large-scale applications. Nevertheless, for certain highly nonlinear evolution models, it is reasonable to expect that the distribution of $\delta u$ might be reasonably close to normal if the number of i.i.d. observations is significant in time and the observation network is sufficiently dense in space. This may happen in assimilation of long time series of satellite observations of ocean surface elevation and temperature, for example.

\section{Effective Inverse Hessian (EIH) method}

\subsection{General consideration}

Here we present a new method for estimating the covariance $V_{\delta u}$ to be used in the case of highly nonlinear dynamics, when $[H(\bar{u})]^{-1}$ is not expected to be a good approximation of $V_{\delta u}$. Let us consider the discretized nonlinear error equation Eq. (5) and denote by $\mathcal{H}$ the left-hand side operator in Eq. (5). Then we can write down the expression for $\delta u$

$\delta u=\mathcal{H}^{-1}\left(V_{\mathrm{b}}^{-1} \xi_{\mathrm{b}}+R^{*}(\bar{u}, \delta u) V_{\mathrm{o}}^{-1} \xi_{\mathrm{o}}\right)$,

whereas for the covariance $V_{\delta u}$ we obtain as follows:

$$
\begin{aligned}
& V_{\delta u}:=E\left[\delta u \delta u^{T}\right]=E\left[\mathcal{H}^{-1} V_{\mathrm{b}}^{-1} \xi_{\mathrm{b}} \xi_{\mathrm{b}}^{T} V_{\mathrm{b}}^{-1} \mathcal{H}^{-1 *}\right]+ \\
& +E\left[\mathcal{H}^{-1} R^{*}(\bar{u}, \delta u) V_{\mathrm{o}}^{-1} \xi_{\mathrm{o}} \xi_{\mathrm{o}}^{T} V_{\mathrm{o}}^{-1} R(\bar{u}, \delta u) \mathcal{H}^{-1 *}\right] .
\end{aligned}
$$

As a result of a series of simplifications described in (Gejadze et al., 2011) the above equation can be reduced to the form

$V_{\delta u} \approx V=E\left[[H(\bar{u}+\delta u)]^{-1}\right]$,

where $H(\bar{u}+\delta u)=V_{\mathrm{b}}^{-1}+R^{*}(\bar{u}, \delta u) V_{\mathrm{o}}^{-1} R(\bar{u}, \delta u)$ is the Hessian of the linearized (auxiliary) control problem. The righthand side of Eq. (11) may be called the effective inverse Hessian $(\mathrm{EIH})$, hence the name of the suggested method. In order to compute $V$ directly using this equation, the expectation is substituted by the sample mean:

$V=\frac{1}{L} \sum_{l=1}^{L}\left[H\left(\bar{u}+\delta u_{l}\right)\right]^{-1}$. 
The main difficulty with the implementation is a need to compute a sample of optimal solutions $u_{l}=\bar{u}+\delta u_{l}$. However, formula Eq. (11) does not necessarily require $u_{l}$ to be an optimal solution. If we denote by $q_{\delta u}$ the p.d.f. of $\delta u$, then equation Eq. (11) can be rewritten in the form:

$V=\int_{-\infty}^{+\infty}[H(\bar{u}+v)]^{-1} q_{\delta u}(v) d v$.

If we assume that in our nonlinear case the covariance matrix $V$ describes meaningfully the p.d.f. of the optimal solution error, then, with the same level of validity, we should also accept the pdf $q_{\delta u}$ to be approximately normal with zero expectation and the covariance $V$, in which case we obtain

$V=c \int_{-\infty}^{+\infty}[H(\bar{u}+v)]^{-1} \exp \left(-\frac{1}{2} v^{T} V^{-1} v\right) d v$

where $c^{-1}=(2 \pi)^{M / 2}|V|^{1 / 2}$. Formula Eq. (12) gives $V$ explicitly, but requires a sample of optimal solutions $u_{l}, l=$ $1, \ldots, L$ to be computed. In contrast, the latest expression is a nonlinear matrix integral equation with respect to $V$, while $v$ is a dummy variable. This equation is actually solved using the iterative process Eq. (19), as explained in the following section. It is also interesting to notice that Eq. (14) is a deterministic equation.

\subsection{Implementation remarks}

Remark 1. Preconditioning is used in variational DA to accelerate the convergence of the conjugate gradient algorithm at the stage of inner iterations of the Gauss-Newton (GN) method, but it also can be used to accelerate formation of the inverse Hessian by the Lanczos algorithm (Fisher et al., 2009 ) or by the BFGS (Gejadze et al., 2010). Since $H$ is selfadjoint, we must consider a projected Hessian in a symmetric form

$\tilde{H}=\left(B^{-1}\right)^{*} H B^{-1}$,

with some operator $B: X \rightarrow X$, defined in such a way that the eigenspectrum of the projected Hessian $\tilde{H}$ is clustered around 1, i.e. the majority of the eigenvalues of $\tilde{H}$ are equal or close to 1 . Since the condition number of $\tilde{H}$ is supposed to be much smaller than the condition number of $H$, a sensible approximation of $\tilde{H}^{-1}$ can usually be obtained (either by Lanczos or BFGS) with a relatively small number of iterations. After that, having $\tilde{H}^{-1}$, one can easily recover $H^{-1}$ using the formula:

$H^{-1}=B^{-1} \tilde{H}^{-1}\left(B^{-1}\right)^{*}$.

Assuming that $B^{-1}$ does not depend on $\delta u_{l}$, we substitute Eq. (15) into Eq. (12) and obtain the version of Eq. (12) with preconditioning:

$V=B^{-1}\left(\frac{1}{L} \sum_{l=1}^{L}\left[\tilde{H}\left(\bar{u}+\delta u_{l}\right)\right]^{-1}\right)\left(B^{-1}\right)^{*}$.
Similarly, assuming that $B^{-1}$ does not depend on the variable of integration, we substitute Eq. (15) into Eq. (14) and obtain the version of Eq. (14) with preconditioning:

$V=B^{-1} \tilde{V}\left(B^{-1}\right)^{*}$,

$\tilde{V}=c \int_{-\infty}^{+\infty}[\tilde{H}(\bar{u}+v)]^{-1} \exp \left(-\frac{1}{2} v^{T} V^{-1} v\right) d v$.

Formulas Eq. (16) and Eq. (17) instead of $H^{-1}$ involve $\tilde{H}^{-1}$ which is much less expensive to compute and store in memory. Let us mention here that the EIH method would hardly be feasible for large-scale problems without appropriate preconditioning.

Remark 2. The nonlinear Eq. (17) can be solved, for example, by the fixed point iterative process as follows

$$
\begin{aligned}
& V^{p+1}=B^{-1} \tilde{V}\left(B^{-1}\right)^{*}, \\
& \tilde{V}=c^{p} \int_{-\infty}^{+\infty}[\tilde{H}(\bar{u}+v)]^{-1} \exp \left(-\frac{1}{2} v^{T}\left(V^{p}\right)^{-1} v\right) d v,
\end{aligned}
$$

for $p=0,1, \ldots$, starting with $V^{0}=[H(\bar{u})]^{-1}$. The iterative processes of this type are expected to converge if $V^{0}$ is a good initial approximation of $V$, which is the case in the considered examples. The convergence of Eq. (18) and other methods for solving equation Eq. (17) are subjects for future research.

Remark 3. Different methods can be used for evaluation of the multidimensional integral in Eq. (18) such as quasiMonte Carlo (Neiderreiter, 1992). Here, for simplicity, we use the standard Monte Carlo method. This actually implies a return to the formula Eq. (16). Taking into account Eq. (15), the iterative process takes the form

$V^{p+1}=B^{-1}\left(\frac{1}{L} \sum_{l=1}^{L}\left[\tilde{H}\left(\bar{u}+\delta u_{l}^{p}\right)\right]^{-1}\right)\left(B^{-1}\right)^{*}$,

where $\delta u_{l}^{p} \sim \mathcal{N}\left(0, V^{p}\right)$. For each $l$, we compute $\delta u_{l}^{p}$ as follows

$\delta u_{l}^{p}=\left(V^{p}\right)^{1 / 2} \xi_{l}$

where $\xi \sim \mathcal{N}(0, I)$ is an independent random series, $I$ is the identity matrix and $\left(V^{p}\right)^{1 / 2}$ is the square root of $V^{p}$. One can see that for each $p$ the last formula looks similar to Eq. (16) with one key difference: $\delta u_{l}^{p}$ in Eq. (19) is not an optimal solution, but a vector having the statistical properties of the optimal solution.

Remark 4. Let us notice that a few tens of outer iterations by the GN method may be required to obtain one optimal solution, while an approximate evaluation of $\tilde{H}^{-1}$ is equivalent (in terms of computational costs) to just one outer iteration of the GN method. One has to repeat these computations $p$ times, however, only a few iterations on index $p$ are required in practice. Therefore, one should expect an order of the magnitude reduction of computational costs by the method 
Eq. (19) as compared to Eq. (16) for the same sample size. Clearly, for realistic large-scale models, the sample size $L$ is going to be limited. Probably, the minimum ensemble size for this method to work is $2 L^{*}+1$, where $L^{*}$ is the accepted number of leading eigenvectors of $V^{p}$ in Eq. (19).

Remark 5. In order to implement the process Eq. (19) a sample of vectors $\varphi_{l}(x, 0)=\delta u_{l}^{p}$ must be propagated from $t=0$ to $t=T$ using the nonlinear model Eq. (1). Therefore, for each $p$ one gets a sample of final states $\varphi_{l}(x, T)$ consistent with the current approximation of $V^{p}$, which can be used to evaluate the forecast and forecast covariance. Since $V^{p}$ is a better approximation of the analysis error covariance than simply $[H(\bar{u})]^{-1}$, one should expect a better quality of the forecast and covariance (as being consistent with $V^{p}$, rather than with $\left.[H(\bar{u})]^{-1}\right)$.

\section{Numerical implementation}

\subsection{Numerical model}

As a model we use the 1D Burgers equation with a nonlinear viscous term:

$$
\begin{aligned}
& \frac{\partial \varphi}{\partial t}+\frac{1}{2} \frac{\partial\left(\varphi^{2}\right)}{\partial x}=\frac{\partial}{\partial x}\left(\mu(\varphi) \frac{\partial \varphi}{\partial x}\right), \\
& \varphi=\varphi(x, t), t \in(0, T), x \in(0,1),
\end{aligned}
$$

with the Neumann boundary conditions

$$
\left.\frac{\partial \varphi}{\partial x}\right|_{x=0}=\left.\frac{\partial \varphi}{\partial x}\right|_{x=1}=0
$$

and the viscosity coefficient

$$
\mu(\varphi)=\mu_{0}+\mu_{1}\left(\frac{\partial \varphi}{\partial x}\right)^{2}, \mu_{0}, \mu_{1}=\text { const }>0 .
$$

The nonlinear diffusion term with $\mu(\varphi)$ dependent on $\partial \varphi / \partial x$ is introduced to mimic the eddy viscosity (turbulence), which depends on the field gradients (pressure, temperature), rather than on the field value itself. This type of $\mu(\varphi)$ also allows us to formally qualify the problem Eqs. (20)-(22) as strongly nonlinear (Fučik and Kufner, 1980). Let us mention that the Burgers equations are sometimes considered in DA context as a simple model describing the atmospheric flow motion.

We use the implicit time discretization as follows

$$
\frac{\varphi^{i}-\varphi^{i-1}}{h_{t}}+\frac{\partial}{\partial x}\left(\frac{1}{2} w\left(\varphi^{i}\right) \varphi^{i}-\mu\left(\varphi^{i}\right) \frac{\partial \varphi^{i}}{\partial x}\right)=0,
$$

where $i=1, \ldots, N$ is the time integration index, $h_{t}=T / N$ is the time step. The spatial operator is discretized on a uniform grid $\left(h_{x}\right.$ is the spatial discretization step, $j=1, \ldots, M$ is the node number, $M$ is the total number of grid nodes), using the "power law" first-order scheme as described in (Patankar, 1980), which yields quite a stable discretization scheme (this

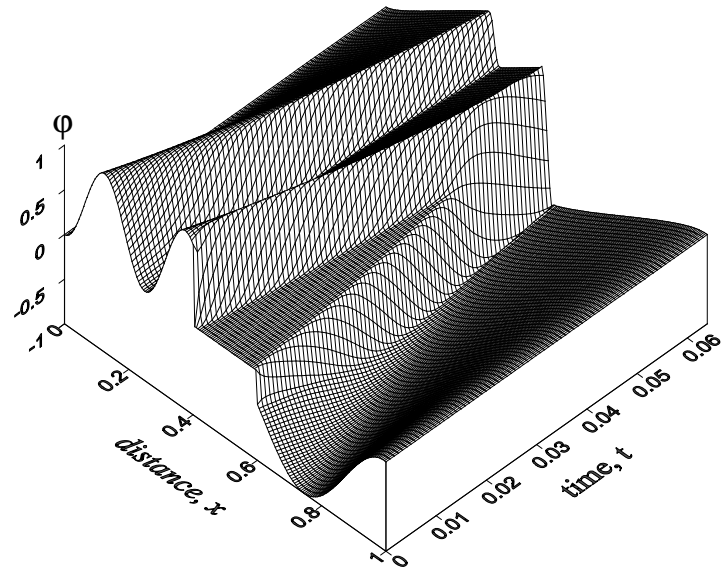

Fig. 2. Field evolution.

scheme allows $\mu(\varphi)$ to be as small as $0.5 \times 10^{-4}$ for $M=$ 200 without noticeable oscillations). For each time step we perform nonlinear iterations on the coefficients $w(\varphi)=\varphi$ and $\mu(\varphi)$ in the form

$\frac{\varphi_{n}^{i}-\varphi_{n}^{i-1}}{h_{t}}+\frac{\partial}{\partial x}\left(\frac{1}{2} w\left(\varphi_{n-1}^{i}\right) \varphi_{n}^{i}-\mu\left(\varphi_{n-1}^{i}\right) \frac{\partial \varphi_{n}^{i}}{\partial x}\right)=0$,

for $n=1,2, \ldots$, assuming initially that $\mu\left(\varphi_{0}^{i}\right)=\mu\left(\varphi^{i-1}\right)$ and $w\left(\varphi_{0}^{i}\right)=\varphi^{i-1}$, and keep iterating until Eq. (23) is satisfied (i.e. the norm of the left-hand side in Eq. (23) becomes smaller than the threshold $\epsilon_{1}=10^{-12} \sqrt{M}$ ). In all the computations presented in this paper we use the following parameters: the observation period $T=0.312$, the discretization steps $h_{t}=0.004, h_{x}=0.005$, the state vector dimension $M=200$, and the parameters in Eq. (22) $\mu_{0}=10^{-4}$, $\mu_{1}=10^{-6}$.

A general property of the Burgers solutions is that a smooth initial state evolves into a state characterized by the areas of severe gradients (or even shocks in the inviscid case). These are precisely the areas of a strong nonlinearity where one might expect violations of the TLH and, subsequently, the invalidity of Eq. (7). For numerical experiments we choose a certain initial condition that stimulates the highly nonlinear behavior of the system; this is given by the formula:

$\bar{u}(x)=\varphi(x, 0)=\left\{\begin{array}{l}0.5-0.5 \cos (8 \pi x), 0 \leq x \leq 0.4, \\ 0,0.4<x \leq 0.6 \\ 0.5 \cos (4 \pi x)-0.5,0.6<x \leq 1 .\end{array}\right.$

The resulting field evolution $\varphi(x, t)$ is presented in Fig. 2.

\subsection{BFGS for computing the inverse Hessian and other details}

The projected inverse Hessian $\tilde{H}(\bar{u}+\delta u)$ is computed as a collateral result of the BFGS iterations while solving the 


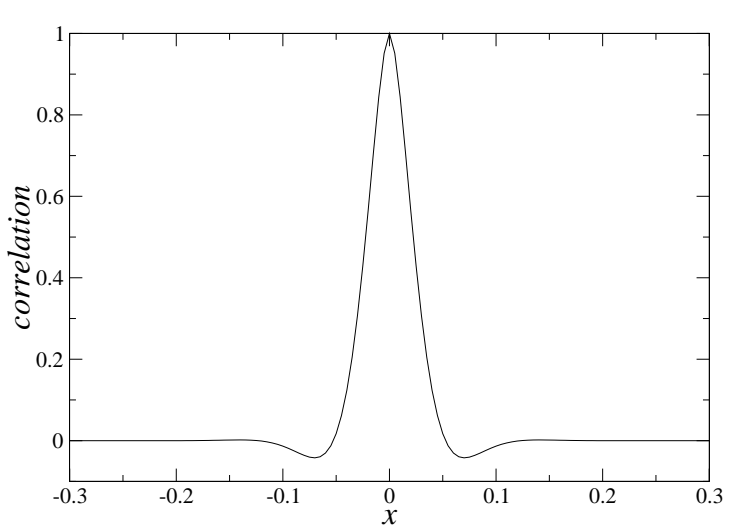

Fig. 3. Correlation function.

following auxiliary DA problem:

$$
\left\{\begin{aligned}
\frac{\partial \delta \varphi}{\partial t}-F^{\prime}(\varphi) \delta \varphi & =0, \quad t \in(0, T) \\
\left.\delta \varphi\right|_{t=0} & =B^{-1} \delta u \\
J_{1}(\delta u) & =\inf _{v} J_{1}(v),
\end{aligned}\right.
$$

where

$$
\begin{aligned}
& J_{1}(\delta u)=\frac{1}{2}\left(V_{\mathrm{b}}^{-1} B^{-1}\left(\delta u-\xi_{\mathrm{b}}\right), B^{-1}\left(\delta u-\xi_{\mathrm{b}}\right)\right)_{X}+ \\
& +\frac{1}{2}\left(V_{\mathrm{o}}^{-1}\left(C \delta \varphi-\xi_{\mathrm{o}}\right), C \delta \varphi-\xi_{\mathrm{o}}\right)_{Y_{o}} .
\end{aligned}
$$

The preconditioner used in our method is

$$
B^{-1}=V_{\mathrm{b}}^{1 / 2}[\tilde{H}(\bar{u})]^{-1 / 2} .
$$

In order to compute $[\tilde{H}(\bar{u})]^{-1 / 2}$ we apply the Cholesky factorization of the explicitly formed matrix $\tilde{H}^{-1}$. However, it is important to note that the square-root-vector product $\tilde{H}^{-1 / 2} w$ can be computed using a recursive procedure based on the accumulated secant pairs (BFGS) or eigenvalues/eigenvectors (Lanczos) as described in (Tshimanga et al., 2008), without the need to form $\tilde{H}^{-1}$ and to factorize it. Consistent tangent linear and adjoint models have been generated from the original forward model by the Automatic Differentiation tool TAPENADE (Hascoët and Pascual, 2004) and checked using the standard gradient test. The background error covariance $V_{\mathrm{b}}$ is computed assuming that the background error belongs to the Sobolev space $W_{2}^{2}[0,1]$ (see Gejadze et al., 2010, for details). The correlation function used in the numerical examples is as presented in Fig. 3, the background error variance is $\sigma_{b}^{2}=0.2$, the observation error variance is $\sigma_{o}^{2}=10^{-3}$. The observation scheme consists of 4 sensors located at the points $\hat{x}_{k}=0.4,0.45,0.55,0.6$, and the observations are available at each time instant.

\section{Numerical results}

First we computed a large sample $(L=2500)$ of optimal solutions $u_{l}$ by solving $L$ times the data assimilation problem Eqs. (1)-(2) with perturbed data $u_{\mathrm{b}}=\bar{u}+\xi_{\mathrm{b}}$ and $y=C \bar{\varphi}+\xi_{\mathrm{o}}$, where $\xi_{\mathrm{b}} \sim \mathcal{N}\left(0, V_{\mathrm{b}}\right)$ and $\xi_{\mathrm{o}} \sim \mathcal{N}\left(0, \sigma_{\mathrm{o}}^{2} I\right)$. This large sample was used to evaluate the sample covariance matrix, which was further processed to filter the sampling error (as described in Gejadze et al., 2011); the outcome was considered as a reference value $\hat{V}^{\circ}$. Then, the original large sample was partitioned into one hundred subsets including $L=25 \mathrm{mem}$ bers and into twenty five subsets including $L=100$ members. Let us denote by $\hat{V}_{L}$ the sample covariance matrix obtained for a subset including $L$ members. Then, the relative error in the sample variance (which is the relative sampling error) can be defined as the vector $\hat{\varepsilon}_{L}$ with the components:

$\left(\hat{\varepsilon}_{L}\right)_{i}=\left(\hat{V}_{L}\right)_{i, i} / \hat{V}_{i, i}^{\circ}-1, i=1, \ldots, M$.

The relative error in a certain approximation of $V$ is defined as a vector $\varepsilon$ with the components:

$\varepsilon_{i}=V_{i, i} / \hat{V}_{i, i}^{\circ}-1, i=1, \ldots, M$.

We compute this error with $V$ in Eq. (27) being estimated by one of the following methods:

1. by the inverse Hessian method, i.e. simply using $V_{\delta u}=[H(\bar{u})]^{-1}$;

2a. by the EIH method implemented in the form Eq. (16), which requires a sample of optimal solutions $\delta u_{l}$ to be computed;

2b. by the EIH method implemented as the iterative process Eq. (19), which requires a sample of $\delta u_{l}$, but does not require that $\delta u_{l}$ are optimal solutions.

For the computation of $V$ by the methods $2 \mathrm{a}$ or $2 \mathrm{~b}$ a sample of $\delta u_{l}$ is required, hence, the result depends on the sample size $L$. The results (obtained by the methods $2 \mathrm{a}$ and $2 \mathrm{~b}$ ) presented in this paper are computed with $L=100$. In the method $2 \mathrm{~b}$ we currently allow enough iterations on the index $p$ for the iterative process Eq. (19) to converge in terms of the distance between the successive iterates. In practice, this requires just a few iterations, typically $2-3$.

In the upper panel in Fig. 4, a set of one hundred vectors $\hat{\varepsilon}_{25}$ is presented in dark lines, and a set of twenty five vectors $\hat{\varepsilon}_{100}$ - in the overlaying white lines. These plots reveal the envelopes for the relative error in the sample variance obtained with $L=25$ and $L=100$, respectively. The graphs of $\varepsilon$ are presented in the lower panel: line 1 corresponds to the method 1 (the inverse Hessian method, see also Fig. 1), lines 2 and 3 - to the methods $2 a$ and $2 b$ (variants of the EIH method). 

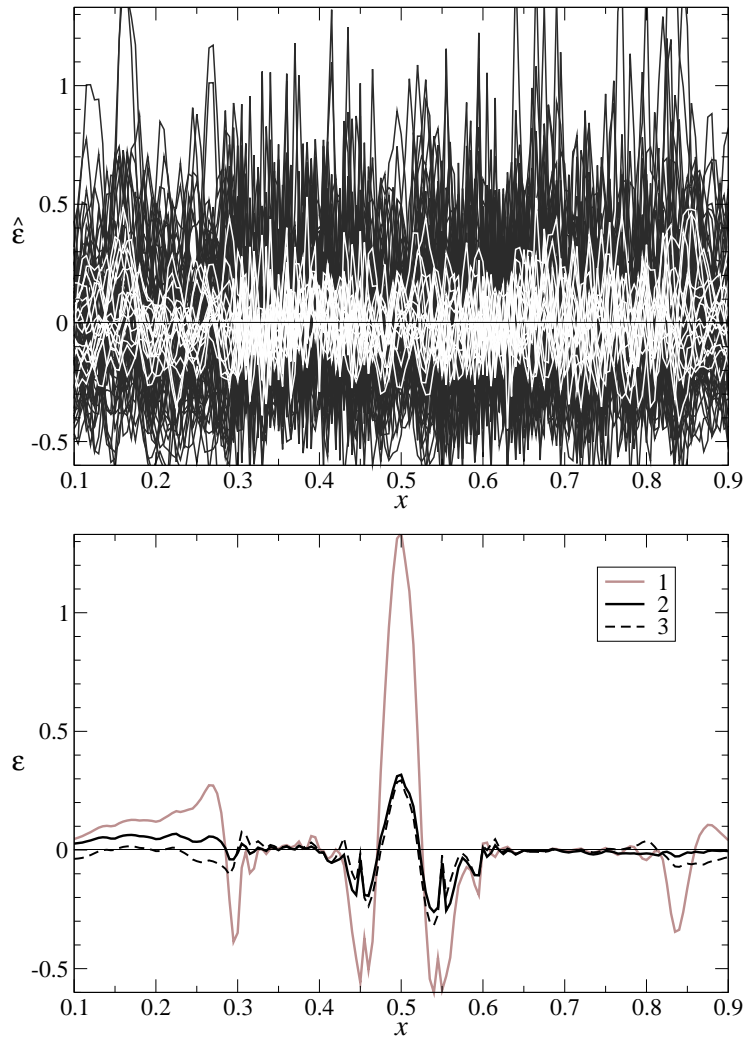

Fig. 4. Up: the sample relative error $\hat{\varepsilon}$. Set of $\hat{\varepsilon}$ for $L=25$ - dark envelope and set of $\hat{\varepsilon}$ for $L=100$ - white envelope. Down: the relative error $\varepsilon$ by the inverse Hessian - line 1, and by the EIH methods with $L=100$ : method 2 a - line 2; method 2 b - line 3 .

Looking at Fig. 4, we observe that the relative error in the sample variance $\hat{\varepsilon}_{25}$ (dark envelope) exceeds $50 \%$ almost everywhere, which is certainly beyond reasonable margins, and $\hat{\varepsilon}_{100}$ (white envelope) is around $25 \%$ (that is still fairly large). In order to reduce the white envelope two times, one would need to use the sample size $L=400$, etc. One should also keep in mind that the relative error in the diagonal elements of the sample covariance matrix is the smallest as compared to its sub-diagonals, i.e. the envelopes for any subdiagonal would be wider than those presented in Fig.4(up). Thus, the development of methods for estimating the covariance (alternative to the direct sampling method) is an important task.

Whereas the method 1 (the inverse Hessian method) gives an estimate of $V_{\delta u}$ with a small relative error (as compared to the sample covariance) in the areas of mild nonlinearity, this error can be much larger in the areas of high nonlinearity. For example, if we imagine that the lower panel in Fig. 4 is superposed over its upper panel, then one could observe line 1 jumping outside the dark envelope in the area surrounding $x=0.5$, i.e. the relative error by the inverse Hessian is significantly larger here than the sampling error for $L=25$.
At the same time, the relative error obtained by the methods $2 \mathrm{a}$ and $2 \mathrm{~b}$ is much smaller as compared to the error in line 1 and it would largely remain within the white envelope. The difference between the estimates by the methods $2 \mathrm{a}$ and $2 \mathrm{~b}$ does not look significant. The best improvement can be achieved for the diagonal elements of $V_{\delta u}$ (the variance). Thus, the covariance estimate by the EIH method is noticeably better than the sample covariance obtained with the equivalent sample size. The suggested algorithm is computationally efficient (in terms of the CPU time) if the cost of computing the inverse Hessian is much less than the cost of computing one optimal solution. In the example presented in this paper one limited-memory inverse Hessian is about 20 30 times less expensive than one optimal solution. Thus, on average, the algorithm $2 \mathrm{~b}$ works about 10 times faster than the algorithm $2 \mathrm{a}$, whereas the results by both the algorithms are similar in terms of accuracy.

\section{Conclusions}

Error propagation is a key point in modeling the large-scale geophysical flows, with the main difficulty being linked to the nonlinearity of the governing equations. In this paper we consider the hind-cast (initialization) DA problem. From the mathematical point of view, this is the initial-value control problem for a nonlinear evolution model governed by partial differential equations. Assuming the so-called tangent linear hypothesis (TLH) holds, the covariance is often approximated by the inverse Hessian of the objective function. In practice, the same approximation could be valid even though the TLH is clearly violated. However, here we deal with such a highly nonlinear dynamics that the inverse Hessian approach is no longer valid. In this case, a new method for computing the covariance matrix, named the "effective inverse Hessian" method, can be used. This method yields a significant improvement in the covariance estimate as compared to the inverse Hessian. The method is potentially feasible for large-scale applications because it can be used in a multiprocessor environment and operates in terms of the Hessian-vector products. The software blocks needed for its implementation are the standard blocks of any existing 4-D Var system. All the results of this paper are consistent with the assumption of a "close-to-normal" nature of the optimal solution error. This should be expected, taking into account the consistency and asymptotic normality of the estimator and the fact that the observation window in variational DA is usually quite large. In this case the covariance matrix is a meaningful representative of the p.d.f. The method suggested may become a valuable option for uncertainty analysis in the framework of the classical 4D-VAR approach when applied to highly nonlinear DA problems. 
Acknowledgements. The first author acknowledges the Russian Foundation for Basic Research and Russian Federal Research Program "Kadry". The second author acknowledges the funding through the Glasgow Research Partnership in Engineering by the Scottish Funding Council. All the authors thank the editor and the anonymous reviewers for their useful comments and suggestions.

Edited by: O. Talagrand

Reviewed by: three anonymous referees

\section{References}

Amemiya, T.: Handbook of econometrics, 1, North-Holland Publishing Company, Amsterdam, 1983.

Courtier, P., Thépaut, J. N., and Hollingsworth, A.: A strategy for operational implementation of 4D-Var, using an incremental approach, Q. J. Roy. Meteor. Soc., 120, 1367-1388, 1994.

Draper, N.R., Smith, H.: Applied regression analysis, 2nd ed., Wiley, New York, 1981.

Fisher, M., Nocedal, J., Trémolet, Y., and Wright, S. J.: Data assimilation in weather forecasting: a case study in PDE-constrained optimization, Optim. Eng., 10, 409-426, 2009.

Fučik, S. and Kufner, A.: Nonlinear differential equations, Elsevier, Amsterdam, 1980.

Gejadze, I., Le Dimet, F.-X., and Shutyaev, V.: On analysis error covariances in variational data assimilation, SIAM J. Sci. Comput., 30, 1847-1874, 2008.

Gejadze, I., Le Dimet, F.-X., and Shutyaev, V.: On optimal solution error covariances in variational data assimilation problems, J. Comput. Phys., 229, 2159-2178, 2010.

Gejadze, I. Yu., Copeland, G. J. M., Le Dimet, F.-X., and Shutyaev, V.: Computation of the analysis error covariance in variational data assimilation problems with nonlinear dynamics, J. Comput. Phys., 230, 7923-7943, 2011.

Hascoët, L., Pascual, V.: TAPENADE 2.1 user's guide, INRIA Technical Report 0300, 78 pp., 2004.
Jennrich, R. I.: Asymptotic properties of nonlinear least square estimation, Ann. Math. Stat., 40, 633-643, 1969.

Le Dimet, F. X. and Talagrand, O.: Variational algorithms for analysis and assimilation of meteorological observations: theoretical aspects, Tellus, 38A, 97-110, 1986.

Lions, J. L.: Contrôle optimal des systèmes gouvernés par des équations aux dérivées partielles, Dunod, Paris, 1986.

Liu, D. C. and Nocedal, J.: On the limited memory BFGS method for large scale minimization, Math. Program., 45, 503-528, 1989.

Marchuk, G. I., Agoshkov, V. I., and Shutyaev, V. P.: Adjoint equations and perturbation algorithms in nonlinear problems, CRC Press Inc., New York, 1996.

Neiderreiter, H.: Random number generation and quasi-Monte Carlo methods, CBMS-NSF Regional Conference Series in Applied Math., 63, SIAM, Philadelphia, 1992.

Patankar, S. V.: Numerical heat transfer and fluid flow, Hemisphere Publishing Corporation, New York, 1980.

Pires, C., Vautard, R., and Talagrand, O.: On extending the limits of variational assimilation in nonlinear chaotic systems, Tellus, 48A, 96-121, 1996.

Rabier, F. and Courtier, P.: Four-dimensional assimilation in the presence of baroclinic instability, Q. J. Roy. Meteor. Soc., 118, 649-672, 1992.

Ratkowsky, D. A.: Nonlinear regression modelling: a unified practical approach, Marcel Dekker, New York, 1983.

Thacker, W. C.: The role of the Hessian matrix in fitting models to measurements, J. Geophys. Res., 94, 6177-6196, 1989.

Tshimanga, J., Gratton, S., Weaver, A. T., and Sartenaer, A.: Limited-memory preconditioners, with application to incremental four-dimensional variational assimilation, Q. J. Roy. Meteor. Soc., 134, 751-769, 2008.

White, H. and Domowitz, I.: Nonlinear regression and dependent observations, Econometrica, 52/1, 143-162, 1984.

Yuan, K.-H. and Jennrich, R. I.: Asymptotics of estimating equations under natural conditions, J. Multivariate Anal., 65, 245260, 1998. 